(1)

CrossMark

\title{
The cancer hypothesis of pulmonary arterial hypertension: are polyamines the new Warburg?
}

\author{
Christopher J. Rhodes \\ Affiliation: National Heart and Lung Institute, Imperial College London, London, UK.
}

Correspondence: Christopher J. Rhodes, NHLI VS Section, Imperial College London, Hammersmith Hospital, Du Cane Rd, London, W12 ONN, UK. E-mail: crhodes@imperial.ac.uk

@ERSpublications

Polyamine metabolism is a new target in pulmonary arterial hypertension also under development in cancer research https://bit.ly/2YnD17o

Cite this article as: Rhodes CJ. The cancer hypothesis of pulmonary arterial hypertension: are polyamines the new Warburg? Eur Respir J 2020; 56: 2002350 [https://doi.org/10.1183/13993003.02350-2020].

Since the identification of elevated anaerobic glycolysis in pulmonary vascular cells from patients with pulmonary hypertension $(\mathrm{PH})$ led to comparisons with the Warburg effect in cancers, $\mathrm{PH}$ has joined the list of cardiovascular and respiratory disorders with a metabolic component to their pathology. Insulin resistance is common and associated with poor outcomes [1], and questions over the importance of prevalent metabolic syndrome remain [2]. Development of libraries of metabolites characterised with high throughput chromatography-mass spectrometry methods has facilitated the screening of large numbers of metabolites in disease tissue samples. The association of alterations in multiple classes of active and breakdown products of metabolism (modified nucleosides, energy metabolites including several long-chain acylcarnitines and tryptophan metabolites in particular) with disease severity [3], survival [4] and right ventricular function [5] has thus been well documented. Therapeutic strategies targeting this are so far in early stages, with one study trialling dichloroacetate to correct glycolytic flux essentially negative, though a post hoc analysis suggested the possibility that genetically susceptible individuals may have shown benefit [6].

In this issue of the European Respiratory Journal, He et al. [7] screened a selected number of metabolites in 30 Chinese patients with idiopathic pulmonary arterial hypertension (IPAH) against matched healthy controls and confirmed elevated spermine in a validation analysis of a further 105 individuals. Spermine was then demonstrated to regulate rat and human pulmonary artery smooth muscle cell (PASMC) proliferation and was elevated in animal models, which were also exacerbated by direct treatment with further spermine. Silencing of spermine synthase, the gene responsible for production of spermine, showed efficacy in preventing PASMC proliferation and development of $\mathrm{PH}$ in the monocrotaline $\mathrm{PH}$ model. Spermine was shown to increase Erk phosphorylation in response to platelet derived growth factor- $\beta$, an effect which was preventable with the protein kinase inhibitor selumetinib, suggesting another potential method of rescue.

Polyamines are required for proliferation and have been shown to be elevated in multiple cancers, with inhibitors of various enzymes in the pathway under investigation as therapeutics. For example the ornithine decarboxylase (ODC) inhibitor difluoromethylornithine (DFMO; ornidyl; eflornithine) is 
approved for treatment of Trypanosome brucei subspecies gambiense and hirsutism and is the focus of multiple cancer clinical trials [8]. Polyamines also regulate ion channels linked to pulmonary arterial hypertension $(\mathrm{PAH})$ such as transient receptor potential $\mathrm{C} 1$, which is reduced in patient whole blood RNA [9] and linked to development of $\mathrm{PH}$ in hypoxic mice [10].

In our previous UK study on metabolomics in $\mathrm{PAH}$ [4] the polyamines 4-acetamidobutanoate and $\mathrm{N}$-acetylputrescine were increased in $\mathrm{PAH}$ patients plasma compared to healthy controls but not independently of bilirubin levels, which was used as a marker of liver function. In this study, spermine levels were not similarly associated with liver function, again assessed by bilirubin, suggesting it may be a less confounded readout of the pathway. 5-methylthioadenosine, another polyamine metabolite produced as a by-product in the synthesis of both spermidine and spermine, was also associated with outcomes (survival) in the UK study, emphasising the strong relationship between this family of metabolites and PAH status (figure 1). In recent transcriptomic analysis of whole blood by microarray [11], RNAseq [9] and lung tissue by RNAseq [12], only one gene was found to be dysregulated in PAH patients across all three studies; AMD1 (encoding adenosylmethionine decarboxylase 1) was consistently lower in PAH. This enzyme catalyses the production of $S$-adenosyl-methionine-amine, which is required for the conversion of the principal polyamine in humans, putrescine, to spermidine (figure 1). The convergence of these multiple avenues of investigation on a single metabolic pathway suggests its pursuit should be prioritised and offers insight into how susceptible patients might be prioritised in any therapeutic strategies developed.

As with other omics strategies, the genomic control of metabolomic profiles is the subject of detailed research [13] and this opens up opportunities to test for causality and association through genetic epidemiology approaches, such as Mendelian randomisation, recently applied to red cell distribution width and iron deficiency in this disease [14]. Refinement of metabolic profiles most informative to diagnosis and risk stratification would facilitate the addition of measurements of these molecules into key clinical management guidelines to bring us towards a biomolecular definition of patient subgroups more likely to respond to targeted therapeutics [15] and for use in trial selection and novel endpoints [16]. Beyond this, we will want to establish which metabolic pathways may provide benefit when therapeutically targeted, and polyamines may be the clearest opportunity for that since dichloroacetate was proposed to correct the Warburg effect.

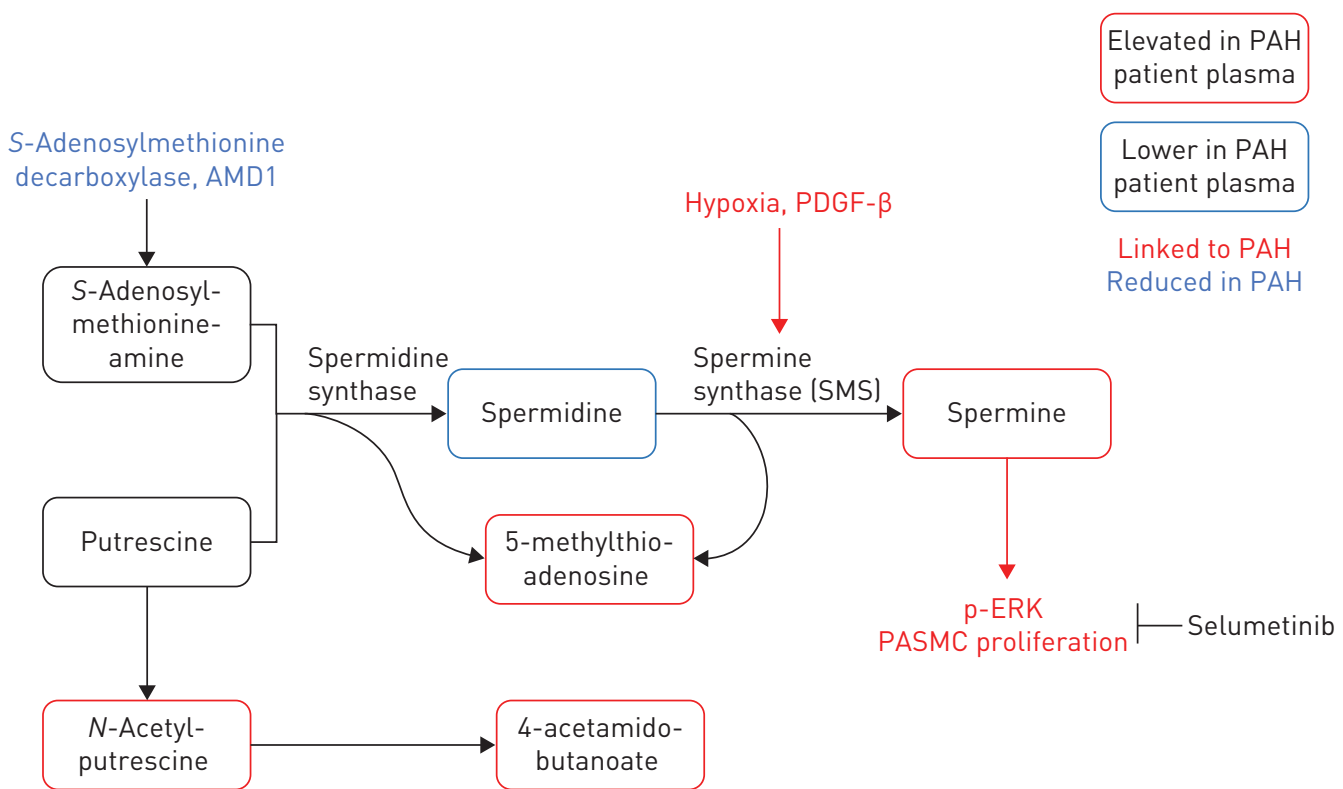

FIGURE 1 Polyamine metabolites linked to pulmonary arterial hypertension (PAH). HE et al. [7] demonstrate the effects of pulmonary hypertension-related stimuli (hypoxia, platelet derived growth factor (PDGF)- $\beta$ ) on spermine synthase (SMS) and the downstream consequences of elevated spermine on extracellular signal-regulated kinase (ERK) and pulmonary arterial smooth muscle cell (PASMC) proliferation, which can be inhibited by the protein kinase inhibitor selumetinib. Other related polyamine metabolites have been demonstrated as elevated and/or associated with survival in PAH patients in other studies (red outlines). 
Conflict of interest: None declared.

\section{References}

1 Zamanian RT, Hansmann G, Snook S, et al. Insulin resistance in pulmonary arterial hypertension. Eur Respir J 2009; 33: 318-324

2 Ussavarungsi K, Thomas CS, Burger CD. Prevalence of metabolic syndrome in patients with pulmonary hypertension. Clin Respir J 2017; 11: 721-726.

3 Lewis GD, Ngo D, Hemnes AR, et al. Metabolic profiling of right ventricular-pulmonary vascular function reveals circulating biomarkers of pulmonary hypertension. J Am Coll Cardiol 2016; 67: 174-189.

4 Rhodes CJ, Ghataorhe P, Wharton J, et al. Plasma metabolomics implicates modified transfer RNAs and altered bioenergetics in the outcomes of pulmonary arterial hypertension. Circulation 2017; 135: 460-475.

5 Brittain EL, Talati M, Fessel JP, et al. Fatty acid metabolic defects and right ventricular lipotoxicity in human pulmonary arterial hypertension. Circulation 2016; 133: 1936-1944.

6 Michelakis ED, Gurtu V, Webster L, et al. Inhibition of pyruvate dehydrogenase kinase improves pulmonary arterial hypertension in genetically susceptible patients. Sci Transl Med 2017; 9: eaao4583.

7 He Y-Y, Yan Y, Jiang X, et al. Spermine promotes pulmonary vascular remodeling and its synthase is a therapeutic target for pulmonary arterial hypertension. Eur Respir J 2020; 56: 2000522.

8 Casero RA Jr, Murray Stewart T, Pegg AE. Polyamine metabolism and cancer: treatments, challenges and opportunities. Nat Rev Cancer 2018; 18: 681-695.

9 Rhodes CJ, Otero-Nunez P, Wharton J, et al. Whole blood RNA profiles associated with pulmonary arterial hypertension and clinical outcome. Am J Respir Crit Care Med 2020; 202: 586-594.

10 Malczyk M, Veith C, Fuchs B, et al. Classical transient receptor potential channel 1 in hypoxia-induced pulmonary hypertension. Am J Respir Crit Care Med 2013; 188: 1451-1459.

11 Elinoff JM, Mazer AJ, Cai R, et al. Meta-analysis of blood genome-wide expression profiling studies in pulmonary arterial hypertension. Am J Physiol Lung Cell Mol Physiol 2020; 318: L98-L111.

12 Stearman RS, Bui QM, Speyer G, et al. Systems analysis of the human pulmonary arterial hypertension lung transcriptome. Am J Respir Cell Mol Biol 2019; 60: 637-649.

13 Long T, Hicks M, Yu HC, et al. Whole-genome sequencing identifies common-to-rare variants associated with human blood metabolites. Nat Genet 2017; 49: 568-578.

14 Ulrich A, Wharton J, Thayer TE, et al. Mendelian randomisation analysis of red cell distribution width in pulmonary arterial hypertension. Eur Respir J 2020; 55: 1901486.

15 Galie N, Channick RN, Frantz RP, et al. Risk stratification and medical therapy of pulmonary arterial hypertension. Eur Respir J 2019; 53: 1801889.

16 Sitbon O, Gomberg-Maitland M, Granton J, et al. Clinical trial design and new therapies for pulmonary arterial hypertension. Eur Respir J 2019; 53: 1801908. 